\title{
Multichannel mapping of fetal magnetocardiogram in an unshielded hospital setting
}

\author{
Donatella Brisinda $^{1}$, Silvia Comani ${ }^{2,3}$, Anna Maria Meloni ${ }^{1}$, Giovanna Alleva ${ }^{3}$, Dante Mantini ${ }^{4}$ \\ and Riccardo Fenici ${ }^{1 *}$ \\ ${ }^{1}$ Clinical Physiology_Biomagnetism Center, Catholic University, Rome, Italy \\ ${ }^{2}$ ITAB-Institute of Advanced Biomedical Technologies, University Foundation 'G. D'Annunzio', Italy \\ ${ }^{3}$ Department of Clinical Sciences and Bio-imaging, Physical Sciences Unit, Chieti University, Italy \\ ${ }^{4}$ Department of Informatics and Automation Engineering, Marche Polytechnic University, Ancona, Italy
}

\begin{abstract}
Objectives To evaluate the feasibility of unshielded in-hospital multichannel mapping of fetal magnetocardiogram (FMCG), with a 36-channel system for standard adult magnetocardiographic (MCG) recordings, and its reliability according to the recommended standards for FMCG.
\end{abstract}

\begin{abstract}
Methods FMCG was ambulatory mapped with a 36-channel MCG system, in six normal pregnancies at different gestational ages. MCG analysis included adaptive digital filtering of $50 \mathrm{~Hz}$, signal averaging, reconstruction of magnetic field distribution (MFD) and source localization. Fixed Point Independent Component Analysis algorithm (FastICA) was used to reconstruct the FMCG, separating them from maternal contamination and noise.
\end{abstract}

\begin{abstract}
Results The quality of FMCG recorded after the 32nd gestational week and reconstructed with FastICA was close to FMCG obtained in shielded rooms, and good enough to measure cardiac intervals and heart rate variability parameters. In two cases, reconstruction of the MFD during the QRS allowed three-dimensional localization of ventricular sources.

Conclusions A first demonstration has been given that multichannel mapping of FMCG can be performed in unshielded clinical environments, with resolution good enough for contactless assessment of fetal cardiac electrophysiology. FastICA processing on unshielded FMCG, recorded after the 32nd week, provided beat-tobeat analysis and heart rate variability assessment. Further work is needed to improve signal reconstruction in early pregnancy. Copyright (c) 2005 John Wiley \& Sons, Ltd.

KEY WORDS: unshielded magnetocardiography; fetal magnetocardiography' independent component analysis; fetal heart rate variability; fetal arrhythmias
\end{abstract}

\section{INTRODUCTION}

Since the first fetal magnetocardiogram (FMCG) was recorded (Kariniemi et al., 1994), this technique has been used for both research and clinical studies of fetal heart function. FMCG is a contactless recording of the magnetic field (MF) produced by the electrical activity of the fetal heart, which is registered outside the maternal abdomen. In contrast to fetal ECG, difficult to obtain because of the insulating properties of the vernix caseosa unless using intrauterine scalp electrode (Hosono et al., 2001), FMCG is a noninvasive procedure that provides information about fetal cardiac electrophysiology as early as the 13th week of gestation. In shielded rooms, the quality of FMCG has reached a resolution sufficient to measure standard cardiac intervals (Leuthold et al., 1999; Horigome et al., 2000; Van Leeuwen et al., 2000;

*Correspondence to: Riccardo Fenici, Biomagnetism CenterClinical Physiology, Catholic University of Rome, Largo A Gemelli, 800168 Rome-Italy. E-mail: feniciri@rm.unicatt.it
Stinstra et al., 2002; Lowery et al., 2003; Grimm et al., 2003a; Comani et al., 2004a) and heart rate variability (HRV) (Van Leeuwen et al., 1999a; Kotini et al., 2001) to detect fetal arrhythmias (Van Leeuwen et al., 1999b; Copel et al., 2000; Wakai et al., 2000; Menendez et al., 2001; Hosono et al., 2002; Comani et al., 2004b), arrhythmogenic risk factors (Menendez et al., 2000; Kahler et al., 2001a; Naheed et al., 1996), other pathological conditions (Horigome et al., 2001; Peters et al., 2001; Kahler et al., 2001b; Quartero et al., 2002), and to characterize the patterns of initiation and termination of reentrant fetal supraventricular tachycardia (Wakai et al., 2003). Recommended standards for FMCG have been recently published (Grimm et al., 2003b).

As for adult magnetocardiography, a major limitation to the clinical use of FMCG has been the high cost of cryogenic recording equipments and magnetically shielded rooms (MSR). Previous attempts to record FMCG outside MSR have been only partially successful (Rassi and Lewis 1995; Baffa et al., 1995; Brake et al., 2001). 
The aim of this pilot study was to evaluate, in accordance with the recommended standards (Grimm et al., $2003 \mathrm{~b}$ ), the feasibility and reliability of multichannel mapping of the FMCG using a system designed for adult magnetocardiography in an unshielded clinical environment (Fenici et al., 2003).

\section{SUBJECTS AND METHODS}

FMCG was recorded, in our unshielded outpatient clinic, with a 36-channel MCG system (CardioMag Imaging Inc) that features 36 LT-DC-SQUIDs (Superconducting QUantum Interference Device) coupled to second-order gradiometers (baseline: $70 \mathrm{~mm}$, pick-up coils: $20 \mathrm{~mm}$ and sensor-to-sensor spacing: $40 \mathrm{~mm}$ ); they have a sensitivity of about $20 \mathrm{fT} / \mathrm{Hz} 1 / 2$ above $10 \mathrm{~Hz}$, and an automatic electronic suppression of the environmental noise, which is sampled with three additional orthogonal SQUID magnetometers. The performance and reliability of the system have been previously validated in experimental and clinical studies (Fenici et al., 2003; Brisinda et al., 2004a,b). In our unshielded setting, fully equipped for cardiac emergency interventions, interferences in the acquisition bandwidth were mainly evident at the frequencies typical of the power-line $(50 \mathrm{~Hz}$ and harmonics). They impeded a reliable identification of the FMCG without additional processing. The peak-topeak noise level was generally between 1.2 and 2 pT. Real-time detection of fetal QRS signals was therefore possible only after digital filtering of the $50 \mathrm{~Hz}$ and its harmonics.

One maternal ECG lead was recorded during FMCG acquisitions. Both MCG and ECG signals were digitally recorded (bandwidth: DC-100 Hz) at $1-\mathrm{kHz}$ sampling rate. The system software tools provide highresolution signal analysis, MF reconstruction, and threedimensional (3D) source localization using the effective magnetic dipole (EMD) model and the current reconstruction (CR) model (Fenici et al., 2003).

\section{STUDY PROTOCOL}

This pilot study aimed at evaluating the feasibility of unshielded FMCG using an adult MCG device, and was ethically approved on a limited number of normal volunteers who gave written informed consent before FMCG acquisitions, in accordance with the recommended guidelines (Grimm et al., 2003b).

Six normal pregnancies were investigated, from the 21 st to the 40th week. Three cases (\#1, \#2, and \#6) were studied more than once, at different gestational ages (Table 1), for a total of 10 FMCG sessions.

FMCG was recorded with the mother lying on the bed and with the flat bottom of the cryostat parallel to it. Special care was devoted to maternal comfort and to avoid the frontal supine position in order to prevent inferior vena cava compression. During FMCG monitoring, the mother was asked to twist slightly oblique to the right or to the left to maximize the identification of fetal
Table $1-$ Characteristics of the study population

\begin{tabular}{lccll}
\hline Case \# & $\begin{array}{c}\text { Mother's } \\
\text { age }\end{array}$ & $\begin{array}{c}\text { Gestational age } \\
\text { (weeks) }\end{array}$ & Position & $\begin{array}{c}\text { Fetal } \\
\text { activity }\end{array}$ \\
\hline 1a & 27 & 21 & Undefined & Undefined \\
1b & 27 & 26 & Undefined & Medium \\
1c & 27 & 29 & Undefined & High \\
2a & 32 & 38 & Vertex & Low \\
2b & 32 & 38 & Vertex & Medium \\
3 & 31 & 39 & Vertex & Medium \\
4 & 34 & 27 & Undefined & Undefined \\
5 & 24 & 40 & Vertex & High \\
6a & 32 & 32 & Vertex & Low \\
6b & 32 & 33 & Vertex & Low \\
\hline
\end{tabular}

QRS peaks. Afterward, fine adjustments of the mother's position were obtained with graded bed movements on the $X-Y$ plane, until both positive and negative components of the fetal QRS field were detected. The position and orientation of the MCG sensors with respect to the fetus were determined using the distances of three tiny light spots projected on the maternal abdomen from fixed (bone) refer points.

A 'scout' recording of $90 \mathrm{~s}$ was performed to check signal quality and correct positioning; finally, a recording of 5 min was performed. In case of three consecutive unsuccessful scouts, FMCG was considered unfeasible. Fetal activity reported by the mother during the recording of FMCG was scored according to guidelines (Grimm et al., 2003b).

\section{SIGNAL ANALYSIS}

FMCG analysis was performed with two different methods. Initially, a simple, real-time approach (method A) was used, based on adaptive digital filtering of $50 \mathrm{~Hz}$, signal averaging triggered by fetal QRS peak, subsequent reconstruction of magnetic field distribution (MFD) and source localization with the EMD and CR models (Fenici et al., 2003). Afterward, the Fixed Point Independent Component Analysis algorithm (FastICA) off-line on raw acquisitions (method B) was applied to separate the fetal signals from maternal contamination and noise, and to reconstruct the time course of fetal cardiac traces (Comon 1994; Bell and Sejnowski 1995; Hyvärinen 1999; Hyvärinen and Oja 2000; Comani et al., 2004c,d; Mantini et al., 2004). ICA permits a completely blind separation of signal sources starting from given mixed input signals under the condition that the mixed traces have components that are independent on one another and are generated from physically distinct sources (Comon 1994; Bell and Sejnowski 1995; Hyvärinen 1999; Hyvärinen and Oja 2000). FMCG recordings fulfill this condition since they result from the summation of the MF variations due to the electrophysiological activity of the maternal and fetal hearts that, although producing similar signals, are separated and beat independently on each other. Background noise is, in general, recognized as the only independent component with a Gaussian distribution among those 
composing the mixed recorded fMCG signals; other noise components, either originating from instrumentation working in the laboratory and its neighborhood or due to fetal movements, were also detected as independent components and, together with background noise, could be disregarded before reconstructing the fetal cardiac signals.

The other ICA constraint that the number of mixed input traces must be greater than the number of required independent components implied that the 36 simultaneous FMCG recordings were grouped in five input clusters of 16 recordings. Each cluster produced one raw fetal trace; therefore, five simultaneous fetal traces were available for the classification of fetal cardiac activity (Comani et al., 2004c,d; Mantini et al., 2004). The reconstructed fetal traces could be smoothed, if necessary, to easy signal interpretation; they were visualized for beats classification and arrhythmia exclusion. Cardiac time intervals were calculated both at a beat-tobeat level and on signal-averaged beats, as reported in the literature (Stinstra et al., 2002; Grimm et al., 2003a, Lowery et al., 2003). The tachogram and HRV, parameters in the time and frequency domains were computed in accordance with standard protocols (Task Force, 1996).

At first, the results obtained with the two methods were compared; afterward, they were used in sequence to obtain (1) a quick evaluation of FMCG quality and optimal sensor positioning with respect to the fetus during signal acquisition (method A); (2) a better signalto-noise $(\mathrm{S} / \mathrm{N})$ ratio of the reconstructed fetal signals for beat-to-beat analysis of cardiac activity (method B).

\section{RESULTS}

Beat-to-beat FMCG analysis was possible in our unshielded setting, only after the 32nd gestational week. In fact, FMCG recorded earlier than 30 weeks (cases \#1 and \#4) had a $\mathrm{S} / \mathrm{N}$ ratio $\leq 0 \mathrm{~dB}$ for average background noise levels ranging from 1.2 to $2 \mathrm{pT}$; therefore, signal quality was insufficient for beat-to-beat analysis, although the QRS was visible after signal reconstruction with FastICA.

An example of reliable beat-to-beat FMCG recording is shown in Figure 1. Fetal QRS was only partly visible from raw signals after adaptive filtering of 50$\mathrm{Hz}$ noise (Figure 1B), but it was clearly detectable on single beats after FastICA processing (Figure 1C) and signal smoothing (Figure 1D), with a marked improvement of the S/N ratio. Averaged QRST morphology of the fetal signals reconstructed with the two methods was identical, although the $\mathrm{S} / \mathrm{N}$ ratio obtained with FastICA and smoothing $(\mathrm{S} / \mathrm{N} \approx 10 \mathrm{~dB}$ on rhythm strips, $\mathrm{S} / \mathrm{N}$ $\approx 22 \mathrm{~dB}$ on averaged beats obtained from 200 cycles) was significantly higher than the values generally associated with simple adaptive filtering of $50 \mathrm{~Hz}(\mathrm{~S} / \mathrm{N} \approx$ $0 \mathrm{~dB}$ on rhythm strips, $\mathrm{S} / \mathrm{N} \approx 12 \mathrm{~dB}$ on averaged beats obtained from 200 cycles) (Figure 1). Atrial activity was not detectable in case \#3 even from averaged waveforms, probably because of significant fetal movements during FMCG recording, which might have produced a larger variability in signal morphology (Zhao and Wakai 2002).

Both positive and negative fetal QRS peaks were simultaneously recorded in cases \#5 and \#6. An example of MF reconstruction during the QRS is shown in Figure 2 (case \#5). 3D localization of ventricular sources at the QRS peak, calculated using the EMD and CR models, was in agreement with the anatomical position of the fetal heart visualized with ultrasound scans. After FastICA processing, the P-wave also became appreciable, and cardiac intervals were measurable not only on selected signal-averaged waveforms (Figure 2A) but also on the entire traces at a single cycle level (Table 2).

Although the $\mathrm{S} / \mathrm{N}$ ratio of unshielded FMCG acquisitions was lower than that usually associated with shielded FMCG recordings $(\mathrm{S} / \mathrm{N} \approx 15 \mathrm{~dB}$ on rhythm strips, $\mathrm{S} / \mathrm{N} \approx 27 \mathrm{~dB}$ on averaged beats obtained from 200 cycles), the quality of fetal signals reconstructed from unshielded FMCG by means of FastICA and smoothing was comparable with that of fetal traces extracted from shielded FMCG (Comani et al., 2004a,b,c,d), and allowed estimating cardiac time intervals on rhythm strips and on averaged beats, as well as HRV parameters (Figure 3, Table 2 and 3).

\section{DISCUSSION}

FMCG is at the moment the most reliable method for noninvasive surveillance of fetal cardiac electrophysiology, especially during the second half of pregnancy,

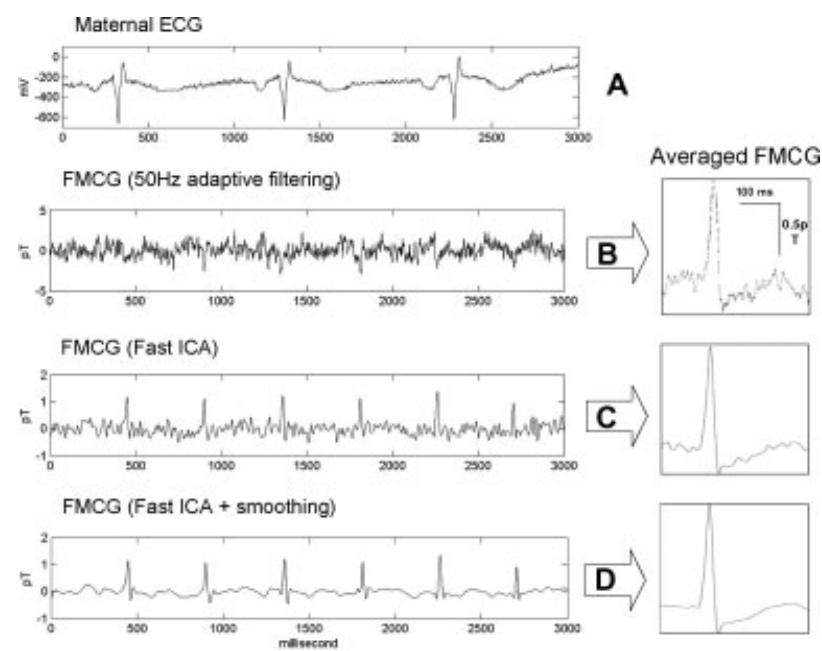

Figure 1-Case \#3, normal pregnancy at 39 weeks. Maternal ECG (A). Fetal QRS complexes were partially appreciable from raw data after adaptive filtering of the $50-\mathrm{Hz}$ noise (B), but clearly identifiable, beat-to-beat, after FastICA (C). Although trace smoothing improved the $\mathrm{S} / \mathrm{N}$ ratio, atrial activity (P-wave) was not detectable in this subject (D). Averaged FMCG morphologies after adaptive filtering of $50 \mathrm{~Hz}$, averaged fetal beats extracted with FastICA and further smoothed are also shown in the right panels. Electrical and magnetic signal amplitudes are given in $\mathrm{mV}$ and $\mathrm{pT}$ respectively; time is measured in milliseconds; the peak-to-peak amplitude of fetal signals is $1 \mathrm{pT}$ on average 

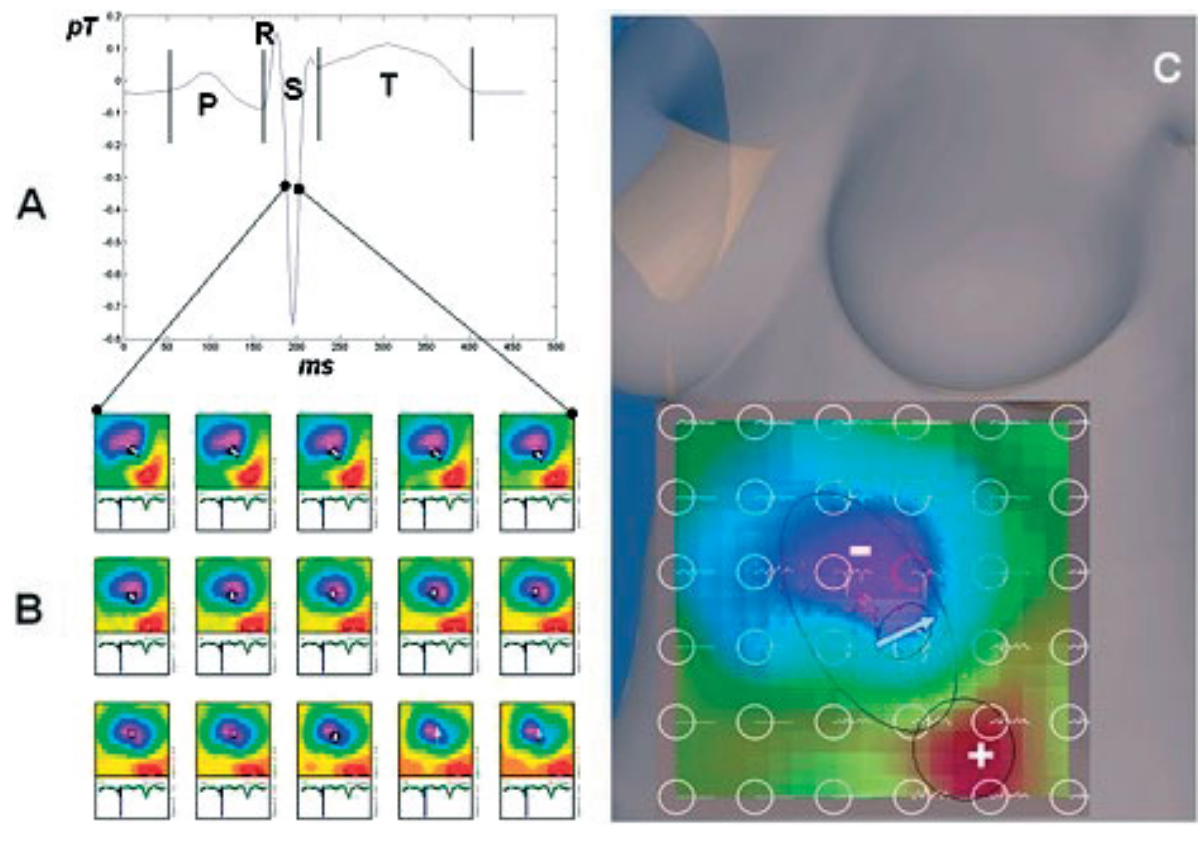

Figure 2-Case \#5, normal pregnancy at 40 weeks. Atrial (P) and ventricular (RS-T) signals are easily identified and measurable from averaged FMCG after processing with FastICA and smoothing (A). The MF distribution during the QRS complex is asymmetrically dipolar and in good agreement with a vertex left occiput anterior position (LOA) of the fetus (B). Both effective magnetic dipole and current reconstruction models provided a reasonable localization of the fetal heart $(\mathrm{C})$. (The mother's torso is a standard 'elastic' torso, which can be shaped to approximate the patient's size, by interactive insertion of her real body measurements. The relative position of the MCG sensors with respect to the fetus is determined by projecting three tiny light spots from the cryostat's bottom onto the mother's abdomen and measuring their distances from fixed (bone) refer points)

Table 2-Cardiac time intervals, estimated beat-to-beat and from averaged FMCG

\begin{tabular}{|c|c|c|c|c|c|c|c|c|c|}
\hline Case \# & Weeks & $\mathrm{N}$ cycles & $\mathrm{RR}$ & P-wave & PQ & QRS & QT & ST & $\mathrm{T}$ wave \\
\hline $2 b$ & 38 & $\begin{array}{c}181 \\
\text { Averaged FMCG }\end{array}$ & $485 \pm 31$ & $69 \pm \underset{66}{ } 11$ & $51 \pm 12$ & $\begin{array}{c}56 \pm 10 \\
57\end{array}$ & $\begin{array}{l}289 \pm 23 \\
295\end{array}$ & $\begin{array}{c}233 \pm 21 \\
238\end{array}$ & $\begin{array}{c}173 \pm 19 \\
166\end{array}$ \\
\hline 3 & 39 & $\begin{array}{c}199 \\
\text { Averaged FMCG }\end{array}$ & $449 \pm 19$ & $\begin{array}{c}59 \pm 14 \\
68\end{array}$ & $\begin{array}{c}43 \pm 10 \\
48\end{array}$ & $\begin{array}{c}56 \pm 7 \\
62\end{array}$ & $\begin{array}{l}299 \pm 18 \\
290\end{array}$ & $\begin{array}{c}239 \pm 17 \\
228\end{array}$ & $\begin{array}{c}171 \pm 13 \\
152\end{array}$ \\
\hline 5 & 40 & $\begin{array}{c}250 \\
\text { Averaged FMCG }\end{array}$ & $372 \pm 22$ & $\begin{array}{c}53 \pm 10 \\
58\end{array}$ & $\begin{array}{c}33 \pm 8 \\
35\end{array}$ & $\begin{array}{c}57 \pm 8 \\
59\end{array}$ & $\begin{array}{l}258 \pm 18 \\
245\end{array}$ & $\begin{array}{c}201 \pm 17 \\
186\end{array}$ & $\begin{array}{c}150 \pm 11 \\
145\end{array}$ \\
\hline $6 b$ & 33 & $\begin{array}{c}216 \\
\text { Averaged FMCG }\end{array}$ & $413 \pm 29$ & $\begin{array}{c}64 \pm 12 \\
53\end{array}$ & $\begin{array}{c}45 \pm 11 \\
43\end{array}$ & $\begin{array}{c}59 \pm 8 \\
56\end{array}$ & $\begin{array}{l}265 \pm 24 \\
271\end{array}$ & $\begin{array}{l}206 \pm 21 \\
215\end{array}$ & $\begin{array}{c}155 \pm 20 \\
156\end{array}$ \\
\hline
\end{tabular}

Cardiac time intervals calculated from averaged FMCG are in italic.

All values are expressed in milliseconds.

(For reference values see: Stinstra et al., 2002; Grimm et al., 2003a; Lowery et al., 2003).

when the presence of electrically insulating vernix caseosa impedes the recording of satisfactory fetal ECG with abdominal leads (Peters et al., 2001). The quality of FMCG recorded with SQUID-based technology in magnetically shielded rooms (MSR) is adequate to identify $\mathrm{P}$ and $\mathrm{T}$ waves of different morphology on a beat-to-beat basis (Comani et al., 2004a,b,c,d; Wakai et al., 2003). However, the high costs and inconvenience of heavy shielding in a clinical environment have prevented a broader diffusion of the method (Brake et al., 2001). Therefore, the challenge is, nowadays, to bring FMCG at the patient's bedside.

This preliminary study aimed at (1) evaluating whether a multichannel system designed for adult MCG (Fenici et al., 2003) could be used to map the FMCG in an unshielded outpatient clinic, providing a signal resolution good enough for clinical use; (2) defining the earliest gestational epoch suitable for the application of the proposed method.

The appropriateness of our unshielded MCG system to detect weak magnetic fields from small hearts had been previously tested in two pilot studies on intact animals of different breeds and size (Brisinda et al., 2004a,b). Our current results demonstrate that FMCG, adequate for the measurement of ventricular time intervals from the averaged QRST waveform in the bandwidth DC-100 Hz, can be recorded in an unshielded hospital environment from the 32 nd gestational week to term. Moreover, we could demonstrate that the time course of fetal signals reconstructed with FastICA from unshielded recordings is suitable to identify atrial activity also and to evaluate all fetal cardiac time intervals and HRV parameters 

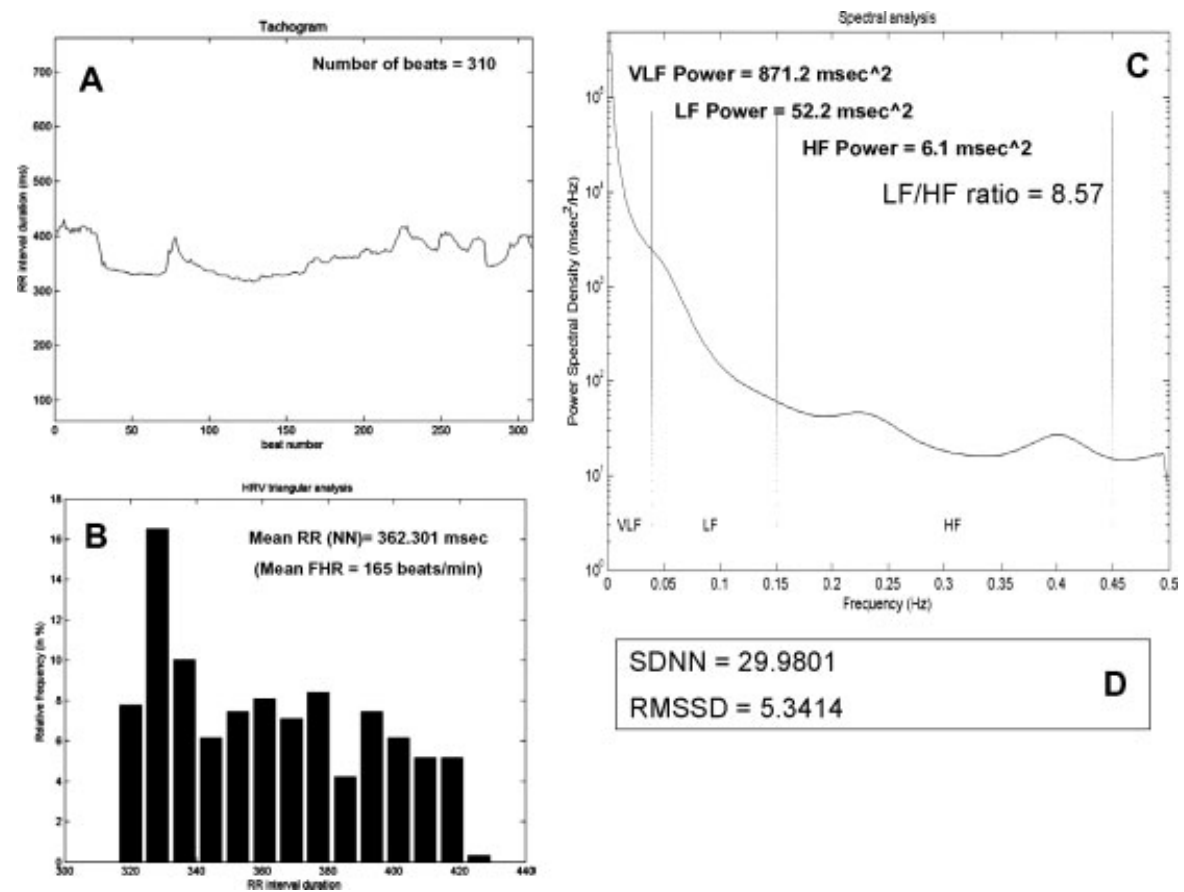

Figure 3-Case \#5, as in Figure 2. Typical automatic report of Fetal HRV analysis obtained on fetal signals reconstructed with FastICA. (A) Tachogram; (B) normal RR (NN) intervals distribution; (C) HRV power spectral density; (D) time-domain parameters. SDNN, standard deviation of all normal sinus RR intervals (in milliseconds); RMSSD, square root of the mean of the sum of differences between adjacent intervals (in milliseconds); VLF, very low frequency; LF: low frequency; HF: high frequency

Table 3-HRV time and frequency domain parameters

\begin{tabular}{lcccccccc}
\hline Case \# & weeks & RR $(\mathrm{ms})$ & SDNN $(\mathrm{ms})$ & RMSSD $(\mathrm{ms})$ & VLF & LF & HF & LF/HF \\
\hline 2b & 38 & 487 & 18 & 11 & 264,17 & 39,3 & 29,95 & 1,31 \\
3 & 39 & 450 & 11 & 8 & 74,36 & 28 & 14,39 & 1,95 \\
5 & 40 & 362 & 30 & 5 & 871,2 & 52,2 & 6,08 & 8,59 \\
6b & 33 & 433 & 23 & 11 & 436,49 & 26,1 & 31,01 & 0,84 \\
Mean & & 433 & 20 & 9 & 411,56 & 36,40 & 20,36 & 3,17 \\
SD & & 52 & 8 & 340,25 & 12,04 & 12,18 & 3,64 \\
\hline
\end{tabular}

(The values of RR and SDNN in this table slightly differ from those of Table 2

(RR column, beat-to-beat values $\pm \mathrm{SD}$ ), because they were calculated from a different number of cardiac cycles).

(Figure 3, Table 2 and 3) according to the recommended standards (Grimm et al., 2003b).

An entire recording session lasted $15 \mathrm{~min}$ on average, with no discomfort for the mother. Online monitoring of fetal QRS peaks permitted the selection of optimal maternal positioning with respect to magnetic sensors, and the consequent recording of both positive and negative fetal MF components. A 90-s scout mapping with subsequent QRS-triggered averaging and MFD reconstruction (Figure 2) allowed confirming the appropriate positioning before the final 5-min recording.

The inverse localization of cardiac sources for ventricular depolarization, performed with the EMD and CR models, was in agreement with the anatomical position of the fetal heart, inferred by trans-abdominal ultrasound scan (Figure 2). To the best of our knowledge, this is the first demonstration that fetal cardiac sources have been localized from unshielded FMCG mapping, which is a powerful adjunct to noninvasive fetal electrophysiological evaluation. In case \#5, MFD associated with ventricular depolarization was reconstructed from data that were averaged after adaptive filtering only, but a significant step forward could be taken from MFD reconstruction after FastICA signal processing. Given the promising results already obtained with FastICA in relation to $\mathrm{S} / \mathrm{N}$ ratio and maps reconstruction from shielded FMCG recordings (Comani et al., 2004d,e), a signal quality adequate for the study of both atrial magnetic fields and ST segment fields can also be expected from the unshielded FMCG (Figure 2). This technical improvement would allow studying fetal MF dynamics during the complete cardiac cycle. The only drawback of FastICA is that it is an off-line procedure. However, it is our opinion that, at present, FastICA is the only method that allows a reconstruction of fetal signals on which beat-to-beat analysis and HRV evaluation is possible from unshielded FMCG. The estimates of fetal cardiac time intervals, calculated on long rhythm strips and on averaged beats (as usually performed in 
other research centers), were comparable with the available age-matched reference values and demonstrated the reliability of the proposed methodology (Stinstra et al., 2002; Grimm et al., 2003a, Lowery et al., 2003). Figures slightly longer than the reference values, but still within the ranges of normal variability, were found for the QT, $\mathrm{ST}$, and $\mathrm{T}$ wave duration in cases \#2b and \#3.

The analysis of HRV in the time and frequency domains has been widely used as a method to study the activity of the autonomic nervous system in adults (Task Force, 1996), although some doubts have been raised about the reliability of the LF $(0.04-0.15 \mathrm{~Hz})$ the HF $(0.15-0.4 \mathrm{~Hz})$ bands, as well as of the LF/HF ratio to unequivocally reflect the sympathovagal balance (Eckberg 1997). Moreover, a direct application of such interpretation to the fetus is questionable.

In our study, normal HRV parameters were found in both time and frequency domains. However, several variables such as gestational age and fetal activity may affect the power density of spectral peaks. In fact, it has been recently reported that association of the conventional HF peak $(0.4 \mathrm{~Hz})$ with respiratory activity in the fetus might be misleading because the frequency of fetal breathing movements is usually between 0.6 and $1 \mathrm{~Hz}$ (Van Leeuwen et al., 2003).

HRV power spectral analysis performed on our data in the bandwidth $0.003-0.45 \mathrm{~Hz}$ has evidenced three different behaviors: in cases \#2 and \#3, the total power (VLF $+\mathrm{LF}+\mathrm{HF}$ components) was in the lower range for the corresponding gestational epochs, with a relative balance between the power of LF and HF components; while in cases \#5 and \#6, the total power values were much higher, with a prevalence of HF components in case \#5 and of LF power density in case \#6. The values of the LF/HF ratio were in agreement with the degree of fetal activity reported by the mother, with the lowest value (case \#6) probably corresponding to a phase of fetal sleep.

\section{LIMITATIONS}

The main limitation of this study was the small number of analyzed cases, but approval was given only to assess the feasibility of unshielded FMCG on few volunteers.

Moreover, although the quality of unshielded FMCG reconstructed with FastICA was close to that obtained in normal age-matched pregnancies investigated in MSR, a critical difference between unshielded and shielded FMCG regarded the gestational epoch for which adequate fetal signals could be extracted. In fact, fetal signals with a resolution sufficient for the quantitative assessment of cardiac intervals and HRV parameters were recorded from the 22nd week in MSR (Comani et al., 2004a,b,d), while in our study, they were recorded only from the 32 nd week. Indeed, no unshielded recordings are available in this study for the 30th and 31st weeks; hence, it cannot be excluded that suitable FMCG might also be recorded for those gestational weeks.

\section{CONCLUSIONS}

Although the quality of previously reported singlechannel unshielded FMCG (Rassi and Lewis 1995; Baffa et al., 1995) was similar to our raw data, we provided the first evidence that multichannel FMCG can nowadays be recorded in an unshielded outpatient clinic by means of commercially available instrumentations of relatively low cost. The $\mathrm{S} / \mathrm{N}$ ratio of recorded $\mathrm{MCG}$ signal and the use of FastICA processing allowed complete quantitative assessment of fetal cardiac activity in four cases recorded after the 32nd gestational week. Although this might obviously limit the clinical application of such procedure to the last $8-10$ weeks of pregnancy, this is clinically important because the presence of the vernix caseosa makes surface fetal ECG recording generally unreliable during that period.

The future challenge will be to improve the resolution of unshielded FMCG in order to set its usefulness in early pregnancy also. As our recordings were performed with a system designed for adult MCG mapping, it is possible that a better sensitivity could be achieved by coupling the implementation of faster noise-reduction software tools based on new mathematical approaches with the development of high-sensitive instrumentation based on low-cost noncryogenic optical magnetometers (Bison et al., 2003) and with a sensor geometry tailored to FMCG applications.

\section{ACKNOWLEDGEMENTS}

Partially supported by MIUR grants \#9906571299_001 and 2001064829_001. The authors are grateful to Dr Carl Rosner, Prof. Alexander Bakharev, Dr Nikolai Korsun, and Dr Karsten Sternickel, for continuous support and technical improvement of multichannel MCG system. The dedication of Mrs. Viola Iacobini, our professional nurse, is outstanding and invaluable.

\section{REFERENCES}

Baffa O, Wakai RT, Sousa PL, Verzola RM. 1995. Fetal heart rate monitoring by magnetocardiograms. Braz J Med Biol Res 28(11-12): $1333-1337$.

Bell AJ, Sejnowski TJ. 1995. Fast blind separation based on information theory. Proceedings of the International Symposium on Nonlinear Theory and Applications, Las Vegas, Nevada, USA; 43-47.

Bison G, Wynands R, Weis A. 2003. A laser-pumped magnetometer for the mapping of human cardio-magnetic fields. Appl Phys 76: $325-328$.

Brake HJM, Ragman AP, Stintra JC. 2001. Fetal magnetocardiography: clinical relevance and feasibility. Proceedings of SQUID Conference. Stenungsbaden: Sweden.

Brisinda D, Meloni Am, Fenici R. 2004a. Contactless magnetocardiographic study of ventricular repolarization in intact Wistar rats: evidence of gender-related differences. Basic Res Cardiol 99(3): 193-203.

Brisinda D, Fenici R, Meloni AM, Fenici P. 2004b. Unshielded multichannel magnetocardiographic mapping of small animals. Biomedizinische Technik 48(2): 162-164.

Comani S, Liberati M, Gabriele E, et al. 2004a. Fetal intra-cardiac intervals for different gestational epochs as evaluated from fetal magnetocardiograms. Biomed Tech 48(2): 150-152. 
Comani S, Liberati M, Mantini D, et al. 2004b. Characterization of fetal arrhythmias by means of fetal magnetocardiography in three cases of difficult ultrasonographic imaging. Pacing Clin Electrophysiol 1647-1655.

Comani S, Mantini D, Pennesi P, Lagatta A, Cancellieri G. 2004c. Independent component analysis: fetal signal reconstruction from magnetocardiographic recordings. Comput Methods Programs Biomed 75/2: 163-177.

Comani S, Mantini D, Lagatta A, Esposito F, Di Luzio S, Romani GL. 2004d. Time course reconstruction of fetal cardiac signals from fMCG: independent component analysis versus adaptive maternal beat subtraction. Physiol Meas 25(5): 1305-1321.

Comani S, Mantini D, Alleva G, Di Luzio S GL, Romani. 2004e. Fetal magnetocardiographic mapping using independent component analysis. Physiol Meas 25(6): 1459-1472.

Comon P. 1994. Independent component analysis - a new concept? Signal Process 36: 287-314.

Copel JA, Liang RI, Demasio K, Ozeren S, Kleinman CS. 2000. The clinical significance of the irregular fetal heart rhythm. Am J Obstet Gynecol 182: 813-817.

Eckberg DL. 1997. Sympathovagal balance: a critical appraisal. Circulation 96: 3224-3232.

Fenici R, Brisinda D, Meloni AM, Fenici P. 2003. First 36-channel system for clinical magnetocardiography in unshielded hospital laboratory for cardiac electrophysiology. Intern J Bioelectromag 5: 80-83.

Grimm B, Kaehler C, Schleussner E, Schneider U, Haueisen J, Seewald HJ. 2003a. Influence of intrauterine growth restriction on cardiac time intervals evaluated by fetal magnetocardiography. Early Hum Dev 74: 1-11.

Grimm B, Haueisen J, Houtilainen M, et al. 2003b. Recommended standards for fetal magnetocardiography. Pacing Clin Electrophysiol 26: 2121-2126.

Horigome H, Takahashi MI, Asaka M, Shigemitsu S, Kandori A, Tsukada K. 2000. Magnetocardiographic determination of the developmental changes in PQ, QRS and QT intervals in the foetus. Acta Paediatr 89: 64-67.

Horigome H, Shiono J, Shigemitsu S, et al. 2001. Detection of cardiac hypertrophy in the fetus by approximation of the current dipole using magnetocardiography. Pediatr Res 50(2): 242-245.

Hosono T, Chiba Y, Shinto M, et al. 2001. A case of complete heart block recorded by magnetocardiography, ultrasonography and direct fetal electrocardiography. Fetal Diagn Ther 16: 38-41.

Hosono T, Kanegawa T, Chiba Y, Kandori A, Tsukada K. 2002. The coincidence of fetal magnetocardiography and direct electrocardiography in a case of fetal atrial flutter due to intracardiac tumor. Fetal Diagn Ther 17: 331-333.

Hyvärinen A. 1999. Fast and robust fixed-point algorithms for independent component analysis. IEEE Trans Neural Netw 10: 626-634.

Hyvärinen A, Oja E. 2000. Independent component analysis: algorithms and applications. Neural Netw 13: 411-430.

Kahler C, Schleussner E, Schneider U, Seewald HJ. 2001a. Prenatal diagnosis of a Wolf-Parkinson-White-syndrome by fetal magnetocardiography (FMCG). BJOG 108: 335-336.

Kahler C, Grimm B, Schleussner E, et al. 2001b. The application of fetal magnetocardiography (FMCG) to investigate fetal arrhythmia and congenital heart defects (CHD) Prenat Diagn 21: 176-182.

Kariniemi V, Ahopelto J, Karp PJ, Katila TE. 1994. The fetal magnetocardiogram. J Perinat Med 2: 214-216.

Kotini PA, Anninos A, Adamopoulos N, Koutlaki G, Galazios P, Anastasiadis A. 2001. Fetal magnetocardiogram recordings and power spectra analysis in biomagnetic arrhythmic signals. J Obstet Gynaecol 21(4): 368-372.

Leuthold A, Wakai RT, Martin CB. 1999. Noninvasive in utero assessment of PR and QRS intervals from the fetal magnetocardiogram. Early Hum Dev 54: 235-243.

Lowery CL, Campbell JQ, Wilson JD, et al. 2003. Noninvasive antepartum recording of fetal S-T segment with a newly developed 151-channel magnetic sensor system. Am J Obstet Gynecol 188: 1491-1497.

Mantini D, Comani S, Pennesi P, Lagatta A, Cancellieri G. 2004. Fetal tailoring of the independent component analysis to multichannel fMCG recordings for an optimal reconstruction of the fetal cardiac signal. Biomed Tech 48(2): 186-188.

Menendez T, Achenbach S, Beinder E, et al. 2000. Prenatal diagnosis of QT prolongation by magnetocardiography. Pacing Clin Electrophysiol 23: 1305-1307.

Menendez T, Achenbach S, Beinder E, et al. 2001. Usefulness of magnetocardiography for the investigation of fetal arrhythmias. Am J Cardiol 88: 334-336.

Naheed Z, Strasburger JF, Deal BJ, Benson DW Jr, Digging SS 1996. Fetal tachyarrhythmia: mechanisms and predictors or hydrops fetalis. J Am Coll Cardiol 27: 1736-1740.

Peters M, Crowe J, Piéri JF, et al. 2001. Monitoring the fetal heart non-invasively: a review of methods. J Perinat Med 29: 408-416.

Quartero HWP, Stinstra JG, Golbach EGM, Meijboom EJ, Peters MJ. 2002. Clinical implications of fetal magnetocardiography. Ultrasound Obstet Gynecol 20: 142-153.

Rassi D, Lewis MJ. 1995. Power spectral analysis of the foetal magnetocardiogram. Physiol Meas 16(2): 111-120.

Stinstra J, Golbach E, Van Leeuwen P, et al. 2002. Multicentre study of fetal cardiac time intervals using magnetocardiography. Brit J Obstr Gynecol 109(11): 1235-1243. and "Fetal Cardiogram Database (update October 02, 2003)" http://bct.tn.utwente.nl/dbhome.htm.

Task Force of the European Society of Cardiology and the North American Society of Pacing and Electrophysiology: Heart rate variability. 1996. Eur Heart J 17: 354-381.

Van Leeuwen P, Lange S, Schüßler M. 2000. Determination of changes in cardiac time intervals and T/QRS ratio during pregnancy using magnetocardiography. In Proceedings of 10th International Conference on Biomagnetism, Aine CJ (ed.). Springer: New York; 581-584.

Van Leeuwen P, Geue D, Lange S, et al. 2003. Changes in the frequency power spectrum of fetal heart rate in the course of pregnancy. Prenat Diagn 23: 909-916.

Van Leeuwen P, Lange S, Bettermann H, Gronemeyer D, Hatzmann W. 1999a. Fetal heart rate variability and complexity in the course of pregnancy. Early Hum Dev 54: 259-269.

Van Leeuwen P, Hailer B, Bader W, Geissler J, Trowitzsch E, Gronemeyer DH. 1999b. Magnetocardiography in the diagnosis of fetal arrhythmia. Br J Obstet Gynaecol 106: 1200-1208.

Wakai RT, Leuthold AC, Cripe L, Martin CB. 2000. Assessment of fetal rhythm in complete congenital heart block by magnetocardiography. Pacing Clin Electrophysiol 23: 1047-1050.

Wakai RT, Strasburger JF, Li Z, Deal BJ, Gotteiner NL. 2003. Magnetocardiographic rhythm patterns at initiation and termination of fetal supraventricular tachycardia. Circulation 107: 307-312.

Zhao H, Wakai RT. 2002. Simultaneity of fetal heart rate accelerations and fetal trunk movements determined by fetal magnetocardiogram actocardiography. Phys Med Biol 47: 839-846. 